\title{
La cultura Embera y las políticas ambientales (Colombia): un análisis interdisciplinar desde la Biología y la Antropología
}

\author{
A cultura Embera e as políticas ambientais (Colômbia): uma análise \\ interdisciplinar desde a Biologia e a Antropologia \\ Embera culture and environmental policies (Colombia): interdisciplinary \\ analysis from Biology and Anthropology
}

\author{
Jair Hernando Castro Romero ${ }^{1}$ \\ Edwin Alexander Sanabria Ospina ${ }^{2}$
}

\begin{abstract}
Resumen
Hoy en día, el estudio académico fragmento al sujeto de estudio para analizarlo desde diferentes perspectivas, así el sujeto presenta variaciones desde los ojos profesionales que lo interpretan y describen. Este trabajo busca realizar un ejercicio pragmático en busca de un diálogo interdisciplinar permanente entre la antropología cultural y las ciencias biológicas, ciencias generalmente aisladas en las estructuras universitarias pero cercanas en disciplinas como sociedad y naturaleza y ecología humana, en este caso se presenta la cultura Embera como modelo de análisis, describiendo tanto sus procesos históricos como su carente situación actual, evidenciando las modificaciones culturales y su relación con la naturaleza, esto con el objetivo de proponer políticas públicas que busquen conservar tanto la cultura como la biodiversidad.
\end{abstract}

Palabras Claves: Embera; Biología; Cultura; Políticas Públicas; Antropología.

\section{Resumo}

Hoje em dia, o estudo acadêmico fragmento o sujeito de estudo para analisá-lo desde diferentes perspectivas, assim o sujeito apresenta variações desde os olhos profissionais que o interpreta e descreve, este trabalho busca realizar um exercício pragmático em busca de um diálogo interdisciplinar permanente entre a antropologia cultural e as ciências biológicas, estas geralmente isoladas nas estruturas universitárias mais vizinhas em disciplinas como sociedade e natureza e Ecologia Humana, Neste caso se apresenta a cultura Embera como modelo de análise, descrevendo desde seus processos históricos até sua carente situação atual evidenciando às modificações culturais e sua relação com a natureza, isto com o objetivo de propor políticas públicas que busquem conservar a cultura como a biodiversidade.

Palavras Chaves: Embera; Biología; Cultura; Políticas Públicas; Antropología.

\begin{abstract}
Nowadays, academic study fragment the subject of study for analyze from different perspectives, subject presents variations from the professional eyes that interprets and describes it, this work seeks to realize a pragmatic exercise from a permanent interdisciplinary dialogue between cultural anthropology and biological sciences, generally isolated in the university structure, but neighboring in disciplines such as society and nature and human ecology. In this case Embera culture is presented as a model of analysis, describing from its historical processes to its present Cultural modifications and their relationship with nature, with the aim of proposing public policies that seek to conserve culture as biodiversity.
\end{abstract}

Key Words: Embera; Biology; Culture; Public policy; Anthropology.

1 Ciencias Biológicas - Ecología y Biodiversidad; Universidad Federal de Integración Latinoamericana UNILA; Foz de Iguazú, Paraná, Brasil; jairhcasrom@gmail.com

2 Antropología y Diversidad Cultural Latinoamericana; Universidad Federal de Integración

Latinoamericana -UNILA; Foz de Iguazú, Paraná, Brasil; edwinsanabria91@ gmail.com 


\section{Contextualización}

Los Emberas son una comunidad indígena colombiana asentada en la selva tropical, un bioma con vegetación exuberante de clima isotérmico y con abundantes precipitaciones (Antolus 2003). Este bioma suele presentar la mayor riqueza mundial, su hábitat está formado por un fauna de loros, tucanes, papagayos, serpientes, ranas, monos, etc. y su flora está constituida por árboles de gran tamaño, con variedades de musgos, enredaderas, orquídeas, lianas, epífitas y saprófitas (Prado 2010), Esta gran diversidad permitió el establecimiento histórico de diferentes grupos indígenas en vastas zonas geográficas, como el caso de los Emberas, (término que significa gente) (Ulloa,2004).

Las relaciones entre términos sociales y biológicos son frecuentes en la realidad social, en este caso la biodiversidad logró conectarse con la cultura, por medio de la representación de sus creencias, como en el totemismo, donde se entreteje una relación simbólica entre la comunidad y algún tipo de animal o vegetal, un ejemplo de esto son los muiscas, pueblo prehispánico que habitó el altiplano cundiboyacense, representandose con la figura del sapo, los cuales elaboraban mitos para la construcción intelectual de su pensamiento, explicando fenómenos como la creación, la maternidad, e inclusive lo inexplicable.

Los emberas con esta conexión cultural también sufren una fuerte presión ambiental, debido a las características de la selva tropical y sus particularidades en el clima, suelo, vegetación y ecofisiología, con un suelo poco profundo, extremadamente pobre en nutrientes, con $\mathrm{pH}$ ácido de 6 , llega solo el $2 \%$ de luz y con un estrato delgado de hojas, semillas, frutos y ramas, que se descompone muy rápidamente inhibiendo la retención de carbono (Ariza, 2008). Esto afecta el ciclo del carbono justo en la fase de aprovechamiento humano, debido a la poca cantidad de nutrientes que hay en el suelo, inhibe el crecimiento de cualquier tipo de cultivo agrícola o comercial. A su vez la vegetación boscosa, presenta una estratificación superior que alcanza los 50 a 55 metros de altura, con troncos esbeltos, corteza delgada, algunos árboles no presentan anillos de crecimiento, mientras que otros presentan raíces aéreas para la sustentación (Antolus 2003). Sumado a lo anterior, la vegetación del interior del bosque es resistente a la baja intensidad de luz a causa de los estratos arbóreos mayores, la polinización frecuentemente se da por aves y murciélagos, mientras que la dispersión se da por diversos tipos de animales, Finalmente la ecofisiología es determinada por una producción elevada y constante de biomasa a lo largo de todo el año (Antolus 2003). Dentro 
de esta vegetación interna suelen estar los cultivos alimenticios, que reciben escasa luz para su manutención, obligando la apertura de espacios forestales con ausencia de árboles de gran porte.

\subsection{Los Emberas}

El pueblo Emberá pertenece al tronco cultural y lingüístico Chocó, conformado por indígenas Waunana y Emberá, originarios del territorio que actualmente corresponde al departamento del Chocó, en el occidente Colombiano (Gonzalez 2011). Los Emberá habitan mayoritariamente entre la cordillera central y el océano Pacífico; cuentan con un gran número de ambientes altitudinales y diferentes comunidades biológicas de las cuales se alimentan, de acuerdo a su territorio, se diferencian entre sí de la siguiente manera: Emberá dobida -gente de río-; Emberá oibida -gente de selva-; Emberá pusabida -gente de mar-, y Emberá eyabida - gente de montaña-. También hay asentamientos humanos Embera en Panamá y Ecuador producto del desplazamiento sufrido por las interacciones socio-ambientales .

Los Eperara Siapidara se reconocen como parte de la gran familia Embera, sin embargo este pueblo presenta una dinámica cultural y de adaptación territorial propia, que se interpreta desde su cosmovisión (UNHCR/Colombia 2011). Su estructura social está constituida sobre la base de una organización familiar de trabajo agrícola, dirige la comunidad la Tachi nawe (nuestra madre), máxima jefe espiritual y sacerdotisa. Su autoridad tradicional está a la cabeza del llamado Jaibana, quién posee el conocimiento del universo mítico ancestral (UNHCR/Colombia 2011).

\subsection{Historia}

Los primeros relatos escritos que se tienen sobre la historia del pueblo Embera en Colombia, se da en el periodo de la colonización española, estos se dividieron en 4 períodos:

I) Denominado "creación de una frontera (1511-1595)", iniciado en 1511 con la fundación varias ciudades entre ellas Santa María la Antigua del Darién y posteriores poblados como Toro, Cáceres, Arma, Anserma, sin relacionarse directamente con los Emberá.

II) "guerra y comercio (1600-1640)" reconocimiento del Rio Atrato por los españoles, iniciando los contactos comerciales, en donde los indígenas adquirían herramientas metálicas.

III) "política misional (1645-1674)", los españoles empezaron a incursionar violentamente sobre el territorio Embera desencadenando reacciones violentas y una mayor introducción del grupo indígena en la selva. Esto condujo a un cambio de táctica por parte de los españoles con la introducción de Misiones Jesuíticas y Franciscanas, que implantaron el 
corregimiento y los castigos. Para los Embera una de las figuras más perseguidas fue el Jaibana, el cual fue protegido por su comunidad negando su presencia y continuando sus trabajos terapéuticos fuera de los ojos de los misioneros y de los agentes occidentales.

IV) “crisis y reconquista (1680-1695)" iniciaron diferentes protestas y levantamientos indígenas siendo disueltas por el ejército español con la ayuda de otros grupos indígenas.

Durante los años (1718- 1730), la fundación de poblaciones en el Alto San Juan y en el Atrato, llevó a la expansión de su territorio hacia el bajo Atrato y la costa Pacífica en 1750 (Ulloa, 2004). En el siglo XIX, la unidad territorial siguió resquebrajándose con la creación de nuevas y diferentes poblaciones en territorio indígena; en el siglo XX, el proceso de fragmentación continúa a causa del frente agroindustrial (caucho, oro, platino, etc.), inmigración de nuevos pobladores; la creación de la prefectura apostólica del Chocó en 1908, las misiones Lauras en 1914 y la instauración de escuelas e internados donde "civilizan" a los indígenas (Ulloa,2004). La realidad política del país también afectó al pueblo Embera, pues la violencia de 1950 contribuyó aún más a la dispersión de esta etnia hacia Panamá y al incremento de la población en el Baudó y costa Pacífica (Ulloa, 2004). Pero esta violencia sigue viva en la actualidad, ya que a partir del 1950 se empieza a generar el conflicto armado en colombia y con el desplazamiento forzado de la comunidad Embera, la cual llega a las principales capitales del país en busca de protección, por parte del estado.

\subsection{Estructura y organización social}

En el tiempo prehispánico los Emberá eran conocidos como indígenas "chocó" o "chocoes", los cuales compartían la misma lengua nativa, cosmovisión y la imagen del Jaibana entre otras características (Ulloa,2004), en la actualidad se subdivide en 4 tribus: Katío, Chamí, Dodiba, y Eperara Siapidara. Dentro de ellos existen los dobida, óibida y eyadiba (Mincultura 2010).

Por otra parte la organización social y de parentesco cumple con la idea de Levis Straus (1969) la cual se a raíz del intercambio de mujeres y objetos con las otras comunidades, buscando una alianza o hermandad con los distintos clanes, evitando enfrentamientos entre pueblos y el incesto, cabe anotar que desde el punto de vista biológico, se busca aumentar la diversidad genética con el fin de disminuir los problemas en la replicación celular, manutención de enfermedades hereditarias, etc.

De esta manera se establece la familia (hombre, mujer e hijos) como unidad básica productora-consumidora y principal escenario de control social y reproducción cultural. Esta vive en un tambo (reunión de varias viviendas) construido por sus miembros, quienes además 
fabrican los instrumentos necesarios para sus trabajos. Las actividades cotidianas se extienden a la parentela, que constituye unidad de cooperación para trabajos más arduos y pesados, como por ejemplo arreglo de terrenos para cultivos, siembra y recolección.

Existe también una división sexual del trabajo, las actividades masculinas son la tumba de monte, la roza, preparación de semillas, siembra, limpieza, cosecha, carga y almacenamiento de los cultivos de plátano, maíz, café, cacao, caña y arroz. también es encargado de las labores económicas del hogar como las transacciones comerciales y el trabajo asalariado. Las actividades femeninas se relacionan con la cosecha, carga y procesamiento de producto para la elaboración de los alimentos. (Ulloa, 2004).

La forma de sanción ante la violación de acuerdos se da en el cepo, el cual es administrado por cada gobierno indígena (Mincultura 2010). Los Emberá reconocen la figura del cabildo como base de su organización política, un órgano público especial, cuya función es representar legalmente a la comunidad, ejercer la autoridad y realizar las actividades que le atribuyen las leyes, sus usos, costumbres y el reglamento interno de cada comunidad, cuyos integrantes son miembros elegidos y reconocidos como una organización sociopolítica tradicional. Sumado a lo anterior, las organizaciones indígenas regionales también representan a los Emberá según su departamento de ubicación, como el Consejo Regional Indígena de Risaralda (CRIR), al CRIDEC (Consejo Regional Indígena de Caldas), a la ORIQUIN (Organización Indígena de Antioquía y a la Organización Regional Indígena del Quindío).

La organización social también está definida bajo el sistema etno-médico, bajo la autoridad del Jaibaná (chamán) significando: Jai que significa espíritu susceptible de curar o causar enfermedad, existiendo entonces lo jais buenos y los jais malos. y la palabra bbaná que significa posesión, es decir, jaibaná significa: el que tiene los espíritus. A diferencia de otras culturas el jaibaná no utiliza yagué (banisteriopsis caapi) ni otros alucinógenos, tampoco tiene origen divino y cualquier persona puede ser jaibaná, según la facilidad que presente para comunicarse con los espíritus. Se les admira porque solucionan problemas pero también se le teme porque tiene la capacidad de hacer el mal (Alcaraz \& Yagarí 2003)

La concepción de salud de la comunidad Emberá entiende que cuando alma y cuerpo están desestabilizados, este último puede exponerse a diversas enfermedades, que pueden ser originadas por seres esenciales (fatales) o las causadas por animales, accidentes caseros y virus (Alcaraz \& Yagarí 2003). Según sea la clase de enfermedad se acude al especialista que la curará, teniendo así al jaibaná, al yerbatero y al hospital respectivamente.

\subsection{Demografía}


Los datos obtenidos en el último censo nacional, muestra que 108.533 personas se auto-reconocen como indígenas Emberá de las cuatro comunidades que integran el grupo. Según el UNHCR/Colombia (Alto comisionado de las Naciones unidas para los refugiados, 2011), 29.094 personas son Emberá Chamí, 37.327 Emberá Dobidá, Embera Katio 38.259 y la población estimada de Eperara Siapidara es de 3.853 personas, ubicados en las regiones, andina, amazónica, atlántica y pacífica.

Según el Departamento Nacional de Estadísticas- DANE- Los Indígenas tienen una base amplia en su pirámide poblacional, donde el $40 \%$ de la población indígena es menor de 15 años, con la más alta fecundidad y dependencia económicamente. Cabe anotar que la tasa de fecundidad global es de 3,85 para mujeres indígenas, mayor que las mujeres de población nacional $(2,44)$. Esta Tasa Global de Fecundidad (TGF) es el número promedio de hijos nacidos vivos que habría tenido una mujer (hipotética) durante su vida reproductiva, si sus años de reproducción hubiesen transcurrido conforme a las tasas específicas de un determinado año y no hubiesen estado expuestas a riesgos de mortalidad hasta el final de su período fértil. (DANE 2005).

\subsection{Vivienda, simbología, abrigo y elementos de uso cotidiano}

Los asentamientos emberas están conformados por viviendas elaboradas de madera, dentro de esta organización se encuentra la casa comunal, en la se establece el cepo y la escuela (Mincultura 2010). Según Ulloa (2004) dentro de la comunidad Embera existen dos modalidades de poblamiento:

I) Caseríos, (habitados por un promedio de 160 personas entre adultos y niños) situándose en las partes altas de los ríos, en el Chocó permanecen casi siempre vacíos, pues sólo en eventos sociales se reúnen todos los habitantes del mismo.

II) Zonas de montaña, éstos permanecen habitados y las casas están rodeadas de chacras con cultivos permanentes, huertos y pequeños semilleros. Los habitantes de estas comunidades viven cotidianamente en viviendas dispersas, comunicándose a través de los ríos y caminos. (Ulloa,2004).

Por otra parte el uso de prendas va estar diferenciado entre hombres y mujeres. El vestuario de la mujer Embera puede llevar faldas de telas coloridas y en la parte superior se utiliza joyería o atuendos especiales para participar en algunas presentaciones o ceremonias programadas durante el año, como es el llamado Paru Wuera (vestido tradicional) en los bailes y ceremonias de Jaibana; el hombre por su parte usa un traje tradicional como lo es el taparrabo, este es para cubrir su parte genital, además de llevar pinturas faciales, tanto 
hombres como mujeres utilizan en sus antebrazos y brazos pinturas con figuras geométricas que identifican símbolos de su cultura. Estos símbolos son importantes debido a que "un símbolo es algo verbal o no verbal, dentro de un lenguaje o cultura particulares que se sitúa en lugar de otra cosa”(Kottat, 2003, p 24 ), esta idea se complementa con la noción de símbolo empleado por Lesli White:

Todo comportamiento humano se origina en el uso de símbolos. Fue el símbolo que transformó a nuestros ancestrales antropoides en hombres y los hizo humanos. Todas las civilizaciones se propagaron y perpetraron solamente por el uso de símbolos... Toda cultura depende de símbolos. Es el ejercicio de la facultad de simbolización que crea la cultura y el uso de símbolos que se torna posible a su perpetuación. Sin el símbolo no habría cultura, y el hombre sería apenas un animal, no un ser humano... El comportamiento humano es el comportamiento simbólico. Un niño de género Homo se vuelve humano solamente cuando es introducido a participar del orden de fenómenos súper orgánicos que es la cultura. Y la llave de este mundo y el medio de participación en el es el símbolo. (White,L. 1995 citado en Laraia,R,2001, p 29).

Es por tanto que los diferentes medio de representación de los símbolos va a demostrar el valor cultural que tienen estes en sus tejidos artesanales, bastones de madera con figuras de animales o seres místicos, los cuales reflejan el totemismo de la comunidad (Ulloa,2004).

Los Embera también elaboran objetos cerámicos (ollas, tazas, copas) y algunos objetos para comercialización (Ulloa,2004). La talla de madera realizadas por los hombres permite la obtención principalmente de machacadores para macerar alimentos, remos, escaleras, palancas para las canoas, bastones para el Jaibana que además es tallado con diversas figuras antropomorfas, juguetes para los niños y balsas con grandes troncos de madera en las zonas de río y en las zonas de montaña elaboran cerbatanas, instrumentos musicales y trapiche (Ulloa,2004).

El tejido de adornos con collares es una actividad principalmente femenina. Hay una variedad de diseños denominados okama, existentes en todas las zonas Emberá, estos son de gran variedad de colores y con diversas figuras (Ulloa,2004).

\subsection{Estrategias de subsistencia, alimentación y economía}

La etnonimia Embera reconoce dos patrones de adaptación eco-cultural, los oibida (gente de selva) y los eyabida (gente de las montañas), cada uno con su propio sistema de obtención de alimentos. El primero está ligado a los recursos selváticos y al binomio plátanomaíz y el segundo, es dependiente del cultivo de caña, con poca producción de plátano y maíz (Rosique 2010). De esta manera la dieta básica de los Embera se compone de plátanos 
verdes cocidos con sal, los cuales se combinan con fríjoles, arroz, maíz, o con alimentos obtenidos del comercio tales como: sardinas, pastas, harinas y galletas, siempre acompañados de carnes o pescados. Además se cuenta con alimentos como el maíz, además de ser un producto base de la dieta Embera está relacionado con actividades de subsistencia y de manutención del conocimiento y cosmovisión.

El sistema económico de los Emberas se ha basado en cultivos tradicionales y en la caza, pesca y recolección (Alcaraz \& Yagarí 2003), la caza y la recolección de frutos se llevan a cabo en el resguardo comunal, es decir tierra que no pertenece a nadie, es un bien común. Cada quien comparte lo obtenido, pues la generosidad y la hospitalidad se encuentran entre los ideales Embera.

Según Ulloa (2004) el cultivo implica un trabajo de tala de los bosques en zonas muy húmedas y quemas en zonas más secas. Sus técnicas de cultivo requieren que la tierra se deje sin cultivar de tres a cinco años. A raíz de esto deben utilizarse nuevos terrenos, pero por falta de tierras en algunas regiones, éstas se cultivan varias veces seguidas. Las agriculturas en los grupos Emberá han sido históricamente de subsistencia, siendo los principales la yuca, plátano, maíz, arroz y frutos de la selva. La principal fuente de proteínas proviene del pescado de rio y en segunda instancia de la caza (Restrepo et al. 2006).

La cacería es una tarea meramente masculina y ha dejado de practicarse por falta de tierras. Los principales animales que se cazan son: Venados, tatabros, pecarís, guaguas, armadillos, ñeques. Las armas más utilizadas son las escopetas y los perros, pues la cerbatana y el arco y flecha están entrando en desuso. Otras carnes consumidas son la de iguanas, cangrejos, tortugas y monos. Dentro de las aves las más predilectas son las perdices, pavas, patos cuervos, guacamayas, golondrinas, palomas, cotorras, loros y tucanes. La dieta de carne se complementa con algunos animales domésticos como patos, gallinas y cerdos, los cuales son cuidados por mujeres.

La pesca se da en zonas de montaña y se encuentra disminuida debido a la alta sobrepoblación y la contaminación generada. Los hombres pescan con atarrayas, arpones, anzuelos, chuzos y barbascos, extrayendo gran variedad de peces como bocachico, dentón, sabaleta, doncella, kicharo, bagre, barbudo, sardina y mojarra. Las mujeres por su parte buscan coroncoras o wakukos, adheridas a las piedras, las cuales atrapan con la mano; Ya los niños participan según su sexo en las actividades. Las mujeres y niñas son las encargadas de limpiarlos y prepararlos.

Los Embera, a través del contacto con la sociedad occidental, han introducido a su cultura una serie de elementos nuevos que tienen que conseguirlos a través del mercado. Así 
tenemos alimentos elaborados industrialmente (galletas, pescado enlatado, dulces, azúcar, pastas, panela, etc.) y utensilios de trabajo (escopetas, cuchillos, hachas, ollas) los cuales son comercializados por los diferentes grupos étnicos que viven en la región. Por su parte los Embera venden madera aserrada y productos elaborados de esta, como los son las canoas o botes; además de elementos propios de la cultura material como canastos, cerámica y adornos.

\subsection{Antropometría}

Para Tobar (1997), el problema más frecuente en los 23 asentamientos analizados, es la desnutrición crónica en los menores de 5 años. Además, se presentaron los niveles más altos de desnutrición (entre 30\% y 50\%) en niños menores a los 18 años dentro de varios grupos indígenas de Colombia, entre ellos los Embera. En promedio el consumo de nutrientes en algunas comunidades Embera, se identificó deficiencia en el consumo total de energía, proteínas, grasa, carbohidratos, fibra, calcio, sodio, vitamina A, tiamina y riboflavina. (Tobar \& chinchilla 2000).

De la misma manera el estudio de Rosique (2010), muestra que la ingesta en las comunidades estudiadas confirma que el promedio de consumo de energía es de 1.578,6 kcal/día, en las que el $50 \%$ de la población no alcanza dicho promedio. Además el 73,4\% de la población presenta deficiencia en la ingesta usual de energía, presentando en las mujeres más deficiencia que en los hombres. Pero esta deficiencia en la ingesta de energía aumentó con la edad de los 0 hasta los 18 años con un pico de aumento a partir de los 9 años en adelante.

Este estudio también observó un aumento de la desnutrición crónica con la edad, de 48,4\% en los niños y niñas de 0-2 años a 76,6\% entre 2 y 5 años y, finalmente a 77,1\% en los jóvenes (10-19 años). A medida que el niño indígena se separa de los cuidados maternos experimenta la sustitución de la leche materna por alimentos de baja calidad nutricional, se profundiza el retraso del crecimiento y el aumento de desnutrición crónica. En otro estudio desarrollado por Restrepo et al. 2006 la prevalencia de desnutrición crónica moderada y grave fue de $63,6 \%$, la desnutrición aguda leve y moderada fue de 8,8\%. La desnutrición crónica y se le ha asociado con las condiciones de pobreza, el hacinamiento y la enfermedad diarreica aguda. La prevalencia de desnutrición crónica, moderada y grave supera a la de los otros grupos indígenas del país y de indígenas del continente. Demostrando el reflejo de las deficiencias acumulativas de alimentación y salud a largo plazo; siendo un indicador de inequidad social, en el que se muestran las condiciones precarias en que se han criado los 
niños y de la asociación de está directamente con el aumento de edad.

La prevalencia de parasitosis es alta, 78,1\%, en donde la infección por parásitos es debida a la alta contaminación fecal del ambiente, la convivencia con animales, los hábitos higiénicos inadecuados y de la falta de suministro de agua potable. El parasitismo es un proceso negativo para el crecimiento y desarrollo de la población infantil porque aumenta la posibilidad de desnutrición al causar inapetencia, competir por los nutrientes, y provocar diarrea o síndrome de malabsorción. (Restrepo et al 2006)

\subsection{Estresores ambientales bióticos y abióticos que afectan la cultura}

La comunidad Emberá posterior a los procesos de colonización y evangelización se vio obligada a restringir su territorio lo que las limitó a dispersarse en diversas comunidades, desembocando en el abandono forzado de las tierras y su reemplazo por territorios de selva húmeda, con suelo, clima y productividad mínima. Estas circunstancias redujeron la disponibilidad de recursos que ofrecía la tierra. Valiéndose de la ceremonia para curar la tierra que se realiza con el propósito de alejar las plagas y los seres que pueden impedir las buenas cosechas. Para la comunidad Embera la presencia de corrientes hidrográficas es un estresor ambiental porque necesita de la corriente del río para desplazarse, pescar y comunicarse. El sistema agrícola depende de la variabilidad climática, limitando la siembra y cosecha de diferentes productos alimenticios (Ulloa, 2004).

Otros problemas se relacionan con el contacto permanente con la cultura occidental, que ha generado una pérdida considerable del conocimiento, usos y costumbres propios. Esto sugiere una presión ambiental capaz de socavar la memoria de las comunidades, impidiendo perpetuar algunas prácticas históricas. Produciendo la migración por grupos o individual a las grandes ciudades del país como Bogotá, Medellín y Cali, que termina en el ejercicio de la mendicidad y de labores distintas a las de la cultura Embera.

El estrés biótico más común es la presencia de mala alimentación debido a la baja calidad de los suelos chocoanos, en donde la producción de alimentos no es eficiente. Cabe anotar que la baja calidad de alimentación también es una consecuencia inmediata de la escasez de recursos económicos ligada a la baja alfabetización y el bajo ingreso per cápita. (Ulloa,2004). Otro estrés ambiental común en Emberá es la prevalencia de parasitosis, según el reporte de la Segunda Encuesta Nacional de Morbilidad realizada en Colombia en 1990 (Restrepo et al 2006). 


\section{Metodología y Objetivo}

El presente trabajo busca fomentar el debate interdisciplinar entre la antropología cultural y las ciencias biológicas, para esto nos apoyándonos en la revisión bibliográfica, la cual nos muestra aspectos particulares sobre la cultura, el medio ambiente y la situación actual de la comunidad indígena Embera en Colombia. La recolección de estos datos bibliográficos se basó en trabajos etnográficos y estudios científicos, elaborados por diferentes autores e instituciones de carácter nacional e internacional.

De esta manera pretendemos generar un diálogo permanente y constructivo, esperando así un mayor análisis que contemple conocimiento multidimensional; enriqueciendo los diferentes aspectos investigativos de cada disciplina, para la elaboración de nuevas políticas públicas que favorezcan la interfase entre cultura y ambiente.

\section{Relación interdisciplinar}

Es evidente la ausencia de políticas públicas que busquen mitigar las presiones socioambientales hacia los pueblos indígenas, así como también faltan esfuerzos para realizar un mayor número de inventarios florísticos y faunísticos para conocer la verdadera diversidad biológica presente en estas áreas, pues países como Colombia, carecen de políticas públicas permanentes hacia la investigación y conservación de biomas.

Desde el punto de vista biológico los animales/plantas suelen ser denominados como especies claves, pues la presencia de ellas modifican los equilibrios dinámicos de las comunidades, es decir, en alguna cultura pueden representante el totemismo por medio del águila, esa será la relación cultural, pero ¿Cuál sería la implicación ecosistemica si el águila no estuviera en esta comunidad?, la respuesta pasa por la modificación del sistema porque las relaciones tróficas se modificarían y algunos niveles tróficos presentarán modificaciones que estarían afectando la manutención de la cultura humana. Por esta razón hay una fuerte relación entre las especies que son representadas en el totemismo y las especies claves que regulan las comunidades.

\section{Conclusión}

A partir de la recolección bibliográfica empleada, podemos constatar que la comunidad Embera desde tiempos colonizadores a la actualidad, se ha visto afectada por las diversas acciones de orden social, cultural y ambiental, generadas principalmentes por diferentes acontecimiento históricos, como lo son: la evangelización, el conflicto armado y la expansión del mercado global, los cuales han llevado en gran parte a la aculturación del 
pueblo Embera y a la destrucción del territorio ancestral, a su vez demuestra el poco interés del estado colombiano, sobre los derechos fundamentales de protección a la vida, la cultura autóctona y su interacción con el medio ambiente.

El análisis de esta problemática contribuye, en primer momento a la importancia del diálogo interdisciplinar entre la antropología cultural y las ciencias biológicas, las cuales permitieron hacer una observación multidimensional por medio de los estudios interdisciplinares, esto solidifica los argumentos y comparte posiciones hacia la creación de posibles políticas públicas en defensa de la cultura y el medio ambiente, en el que habitan la comunidades Emberas en Colombia.

\section{Agradecimientos}

La revisión bibliográfica fue realizada para la disciplina de Ecología humana con la participación de Licet Calambas y Fares Restrepo, agradecemos por su compromiso y a la Banca del seminario latinoamericano de cultura por sus sugerencias.

\section{Referencias}

ALCARAZ, L. G-M \& YAGARI, T. L. La concepción de la curación chamánica entre los indígenas Emberá de Colombia: Un proceso de comunicación socio-cultural y fisiológica. Revista investigación y educación en enfermería. Universidad de Antioquia,(21-2):p.6078,2003.ISSN 01205307-22160280. Disponible en http://bibliotecadigital.udea.edu.co/dspace/handle/10495/5020. Acesso en : 29 jul.2017.

ANTOLUS. Selva. Disponible en: https://antolus2003.files.wordpress.com/2012/09/selva1.pdf

Acceso en: 20 de Julio de 2017

ARIZA, F. La selva tropical. Disponible en: http://ocw.um.es/ciencias/geobotanica/Material\%20de\%20clase/Teoria/tema22.pdf. Acceso en : 20 de Julio de 2017.

Departamento Administrativo Nacional de Estadística (DANE). La visibilización estadística de los grupos étnicos colombianos. Imprenta nacional. Disponible en: www.dane.gov.co/files/censo2005/etnia/sys/visibilidad_estadistica_etnicos.pdf . Acceso en: 20 de Julio de 2017.

GONZÁLEZ, H. La ablación genital femenina en comunidades Emberá chamí. Cadernos Pagu. n.3, p. 163-183. Jul-Dic.2011.ISSN 1809-4449. Disponible en http://www.scielo.br/scielo.php?script=sci_arttext\&pid=S0104-83332011000200006. Acceso 
30 de Jul de 2017.

KOTTAK,C. Espejo para la humanidad: introducción a la antropología cultural. Madrid: Mcgraw-hill, 2003. 338 p.

LARAIA, R. Cultura um conceito antropológico. Rio de Janeiro: Zahar Ed, 2001.117 p.

Ministerio de Cultura de Colombia. Embera Chamí. 2010. Disponible en: http://www.mincultura.gov.co/areas/poblaciones/noticias/Documents/Caracterizaci\%C3\%B3n \%20del\%20pueblo\%20Embera\%20Cham\%C3\%AD.pdf. Acceso en: 20 de Julio de 2017.

PRADO,C. Qué son los Biomas? http://www.uv.mx/personal/tcarmona/files/2010/08/Prado-.pdf. Acceso en: 20 de Julio de 2017

RESTREPO B.N,;. M.T, BELTRÁN. J.C, RODRIGUEZ M, RAMIREZ. M.E. Estado nutricional de niños y niñas indígenas de hasta seis años de edad en el resguardo EmberaKatío, Tierralta, Córdoba, Colombia. Biomedica 26, p. 517-27, 2006.

ROSIQUE G.J, RESTREPO C, M.T, MANJARRÉS C, L.M, GÁLVEZ A, \& SANTA M, J. Estado nutricional y hábitos alimentarios en indígenas Embera de Colombia. Revista chilena de nutrición, 37(3), p. 270-280, 2010.

STRAUSS,L. Las estructuras elementales del parentesco. Buenos Aires, Editora Paidos, 1969. $575 \mathrm{p}$.

TOBAR, V. L-F. \& CHINCHILLA, M.M. Aspectos nutricionales y alimentarios de las comunidades indígenas colombianas. Disponible en: http://www.banrepcultural.org/blaavirtual/geografia/geofraf1/aspectos.htm. Acceso en: $21 \mathrm{de}$ Julio de 2017.

TOBAR L, CHINCHILLA M, CORTÉS \&, MONDRAGÓN A. Estado nutricional de las comunidades indígenas y poblaciones afrocolombianas. Bogotá: Pontificia Universidad Javeriana,1997. 234 p.

ULLOA, E. Grupo indígena los Embera. Disponible en http://www.banrepcultural.org/blaavirtual/geografia/geograf/embera1.htm . Acceso en: 20 de Julio de 2017. 